\title{
Alternating Magnetic Field Losses in ATLAS Type of Aluminum Stabilized NbTi Superconductors
}

\author{
E. W. Boxman ${ }^{2}$, A. Nijhuis ${ }^{1}$ and H. H. J. ten Kate ${ }^{2}$ \\ ${ }^{1}$ University of Twente, Low Temperature Division, \\ 7500AE Enschede, The Netherlands \\ ${ }^{2}$ European Organization for Nuclear Research (CERN), \\ Geneva 23, CH-1211, Switzerland
}

\begin{abstract}
During ramping up- and down of the current in large-scale magnets the ramp losses are an important factor affecting the thermal and electro-magnetic stability of the system. The calculation of the losses is not straightforward due to the large dimensions of the conductor $\left(\sim 600 \mathrm{~mm}^{2}\right)$ implying that diffusion effects have to be taken into account. The AClosses of the Al stabilized NbTi cable conductors used in the ATLAS magnet system were measured in $0.5 \mathrm{~m}$ long samples, using an inductive method with pick-up coils as well as the calorimetric method. External varying magnetic fields up to 2 tesla amplitude were applied parallel and perpendicular to the conductor wide surface. The results are compared to theory. It is found that hysteresis loss, eddy current loss in the Aluminum cladding and cable-to-cladding coupling loss contribute most to the AC loss.
\end{abstract}

\section{INTRODUCTION}

Despite the zero resistance of superconductors, alternating current and alternating magnetic field will cause energy dissipation (AC losses) in the superconductors. Energizing the three toroids and central solenoid in the ATLAS magnet system [1] consequently means that generated heat has to be removed by cooling and the temperature margin guaranteeing thermal and electro-magnetic stability of the conductors must be controlled. In low-temperature superconductors, theory on AC loss is quite well understood, but still precise numbers are difficult to attain due to uncertainties in many conductor parameters. In the case of large size Al stabilized conductors, like in ATLAS, the calculations are strongly influenced by the magnetic field dependent resistivity of the pure Aluminum. Often the best solution is to measure the loss. Here, AC-loss measurements on short samples of the Barrel Toroid and the Central Solenoid conductors and a comparison to theory are presented. Details of the conductors are in reference [1]. Here it suffices to recall the main characteristics. The Barrel Toroid coductor has overall dimensions of the Aluminum (pure, with $\mathrm{RRR}=1500$ or more at $0 \mathrm{~T}$ ) of $57 \times 12 \mathrm{~mm}^{2}$ and it contains a 38 strands Rutherford cable with dimensions of $25 \times 2.3 \mathrm{~mm}^{2}$, positioned in the center (see figure 1). 


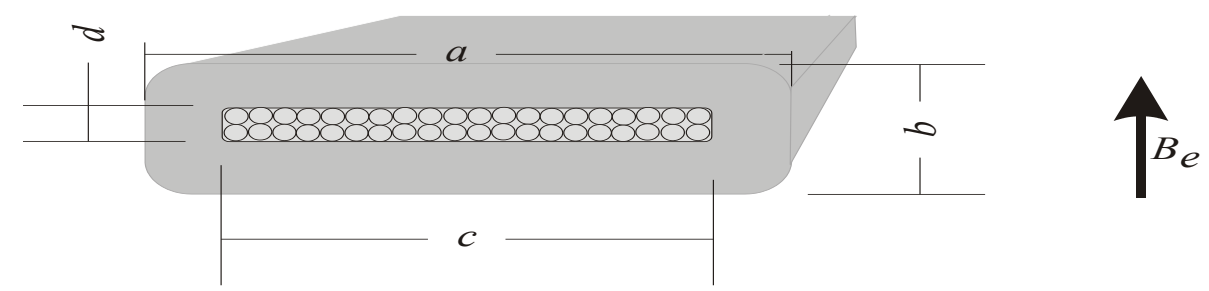

FIGURE 1. Large size Aluminum stabilized $\mathrm{NbTi} / \mathrm{Cu}$ superconductor for the Barrel Toroid, sizes $57 \times 12 \mathrm{~mm}^{2}$, Ic is $65 \mathrm{kA}$ at $5 \mathrm{~T}$. Here the magnetic field direction is shown perpendicular to the conductor.

The critical current is $65 \mathrm{kA}$ at $5 \mathrm{~T}$ while the conductor operates at $20.5 \mathrm{kA}$ in a magnetic peak field of $3.9 \mathrm{~T}$ in the coil windings. The Solenoid conductor is similar but much smaller, $30 \times 4.25 \mathrm{~mm}^{2}$ and is used at $7.8 \mathrm{kA}$ in a $2.3 \mathrm{~T}$ peak field.

The underlying importance is to predict the energy dissipation of the entire ATLAS magnet system under operating conditions. The measurements are performed with linear increasing magnetic fields at different ramp rates. The measurements were performed in the AC loss test facilities at the University of Twente in the Netherlands [1,2].

\section{AC-losses in Aluminum Stabilized Superconductors without Transport Current}

In large size Aluminum stabilized $\mathrm{NbTi} / \mathrm{Cu}$ Rutherford type of cables (see figure 1), five different contributions to AC-losses can be distinguished: (1) Hysteresis loss; (2) Interfilament Coupling current loss in the individual strands (IFCC); (3) Interstrand Coupling current loss (ISCC) in the Rutherford cable; (4) Eddy current loss in the Aluminum stabilizer; and (5) Coupling current loss between the Aluminum Cladding and the Rutherford cable (CCCC). Transport current loss is not taken into account, since in the ATLAS magnets the operating current less than $1 / 3$ of the critical current.

\section{Hysteresis Loss}

During a magnetic field cycle, energy is required to drive the flux inside the superconductor. This hysteresis loss $Q_{h y s}$ is proportional to the filament diameter $a_{f i l}$ and the critical current density $J_{c}$. For magnetic field variations $B_{m}$ much larger than the penetration field $B_{p}$, the loss per cycle is given by [3]:

$$
Q_{\text {hys }}=\frac{8 \cdot n_{\text {fil }} \cdot n_{\text {str }} \cdot n_{\text {tum }} \cdot l_{\text {tum }} \cdot a_{\text {fil }}^{B i \max }}{3 \pi} \int_{B i \min }^{\ln } J\left(\overline{B_{m}}\right) d \overline{B_{m}} \quad[\mathrm{~J} / \text { cycle }],
$$

where $J_{c}(B)$ is the critical current density, $n_{f i l}$ the number of filaments, a $a_{i l}$ the area of a filament, $n_{s t r}$ the number of strands, and $l_{\text {turn }}$ the length of one turn that sees the magnetic field sweep with peak value $B_{m}$. In equation (1), $B_{i \max }$ and $B_{i \min }$ are the maximum and minimum internal values of the magnetic field respectively. They depend on the field rate and so the hysteresis loss is depending on the field rate.

\section{Eddy current loss in the Aluminum cladding}

When the skin depth is large compared to the dimensions of the composite, the screening currents flow through the whole composite. In the case of a steady $d B_{e} / d t$ where the time $T_{m}$ for the field to rise to $B_{m}$ is large compared to the characteristic time constant the loss per unit volume per cycle is: 


$$
Q_{e}=\frac{B_{m}^{2}}{2 \mu_{0}} \frac{8 \tau}{T_{m}}
$$

When using the electrical moment of inertia $J_{m y}$ and at low frequency, the power loss per unit volume can be expressed as [4]:

$Q_{e}=\int_{c y c l e} P_{e} d t=\int_{c y c l e} \frac{a b^{3}}{12} \frac{\dot{B}_{i}}{\rho_{a l}} \frac{1}{a b} d t=\int_{-T_{m}}^{T_{m}} J_{m y} \frac{B_{m}^{2}}{\rho_{a l} T_{m}^{2}} \frac{1}{a b} d t=J_{m y} \frac{B_{m}^{2}}{\rho_{a l} T_{m}} \frac{2}{a b} \quad\left[\mathrm{~J} / \mathrm{cycle} / \mathrm{m}^{3}\right]$,

where $\rho_{a l}$ is the resistivity of the Aluminum cladding. Combining (2) and (3) yields an expression for the characteristic time constant:

$$
\tau=\frac{\mu_{0}}{2 a b} \frac{J_{m y}}{\rho} .
$$

When the composite is exposed to an external magnetic field $B_{e}$, changing at rate $d B_{\mathrm{e}} / d t$, the internal magnetic field is different as screening currents expel the external field in opposite direction. Assuming the field inside the composite is $B_{i}$ one finds [5]:

$$
B_{i}=B_{e}-\tau \dot{B}_{i}
$$

When ramping the magnetic field (up and down with constant $\mathrm{dB} / \mathrm{dt}$ ), the solution is:

$$
B_{i}(t)=\left(\frac{B_{m}}{T_{m}}\right) \cdot\left[\left(t-n T_{m}\right)-\tau\left(1-\exp \left(-\left(t-n T_{m}\right) / \tau\right)\right)\right]+B_{i \min } \quad[\mathrm{T}],
$$

where $B_{i \min }$ is the minimum internal field. In figure 2, the effect is shown for an oscillating magnetic field varying around $B=0 \mathrm{~T}$ with constant ramp rate. Differentiation of (6), substitution in $P_{e}$ and integration over one cycle yields the loss per cycle per unit volume, $Q_{e}$ :

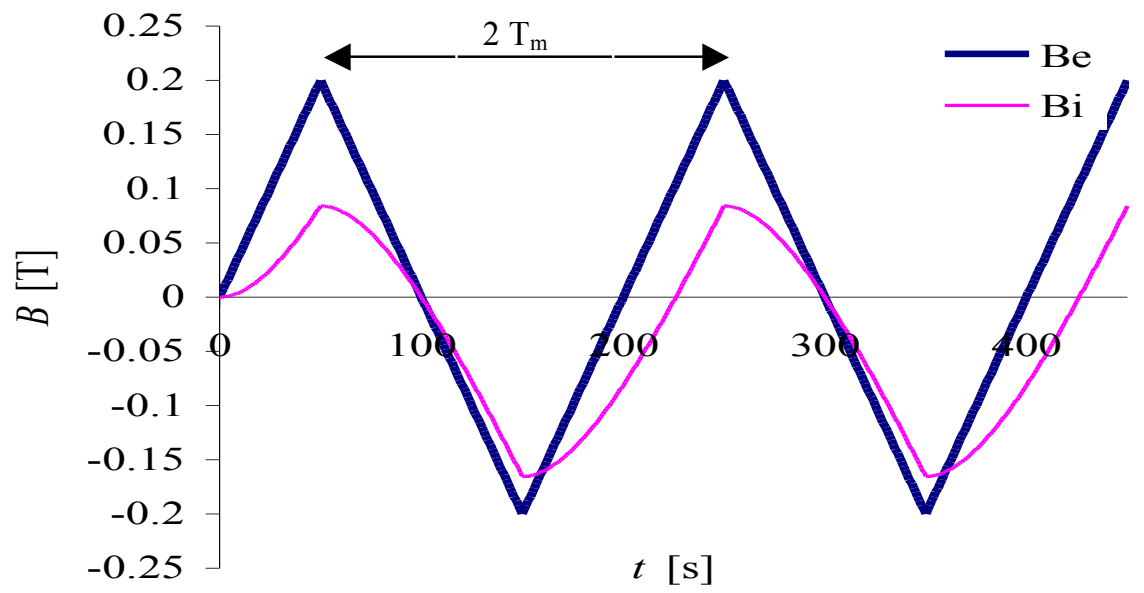

FIGURE 2. The external (applied) $B_{e}$ and internal (calculated) magnetic field $B_{i}$ as a function of time described by equation 6. Part of the external magnetic field is shielded. Ramping up and down the field with $0.1 \mathrm{~s}$ ramping time, results in a considerably reduced peak-to-peak value of the magnetic field as compared to the external magnetic field. In the figure, the time constant $\tau=41 \mathrm{~s}$ with reference to a period time $2 \mathrm{~T}_{\mathrm{m}}$ of $0.2 \mathrm{~s}$. 


$$
Q_{e}=\frac{4 \tau}{\mu_{0}}\left(\frac{B_{m}^{2}}{T_{m}}\right) \cdot\left[1-\frac{3 \tau}{2 T_{m}}\left(1+\frac{1}{3} \exp \left(-2 T_{m} / \tau\right)-\frac{4}{3} \exp \left(-T_{m} / \tau\right)\right)\right] \quad\left[\mathrm{J} / \text { cycle. } \mathrm{m}^{3}\right]
$$

In pure metals the resistivity strongly depends on the magnetic field up to about $0.3 \mathrm{~T}$. Therefore, this calculation is not accurate at low magnetic fields as the time constant $\tau$ is also changing with magnetic field. When the time constant decreases the internal magnetic field $B_{i}$ will follow the external magnetic field more closely. This is the case for alloys or in current paths with highly resistive sections. Note that this kind of coupling loss is also present in BSCCO high temperature superconductors usually having a silver (-alloy) cladding.

\section{Cable-Cladding Coupling Currents (CCCC)}

In a standard Rutherford cable, under AC conditions the induced currents will follow a path directly from strand to strand and the contact resistance between strands has to be calculated to find the associated loss. However, in ATLAS cables, the currents can take a low-resistivity path through the Aluminum as shown schematically in figure 3 . Not only the resistivity of Aluminum is considerably lower than of copper, but also the electrical connection between Aluminum and strands (inermetallic bonding) is much better than the electrical connections between individual strands in the cable. The new current loop can be described using an effective resistivity. The current path is similar to the eddy current path of an Aluminum bar with the difference that the area is as twice as small. The loss is proportional to the square of the area, i.e. for the current path shown in figure 3, the loss is a factor 4 less. As this considers only half of the conductor, the total loss is half the eddy current loss of an ordinary Aluminum bar. The loss per meter is independent of the twist pitch. This kind of Cable-Cladding Coupling Currents will be referred to as CCCC's. The loss per cycle and per unit volume is:

$$
Q_{c c c c}=\frac{2 \tau_{c c c c}}{\mu_{0}}\left(\frac{B_{m}^{2}}{T_{m}}\right) \cdot\left[1-\frac{3 \tau_{c c c c}}{2 T_{m}}\left(1+\frac{1}{3} \exp \left(-2 T_{m} / \tau_{c c c c}\right)-\frac{4}{3} \exp \left(-T_{m} / \tau_{c c c c}\right)\right)\right],
$$

with:

$$
\tau_{c c c c}=\tau=\frac{\mu_{0}}{2 a b} \frac{J_{m y}}{\rho_{e f f}}
$$

where $\rho_{\text {eff }}$ is the effective resistivity of the current path [6]. In the case the electrical con-

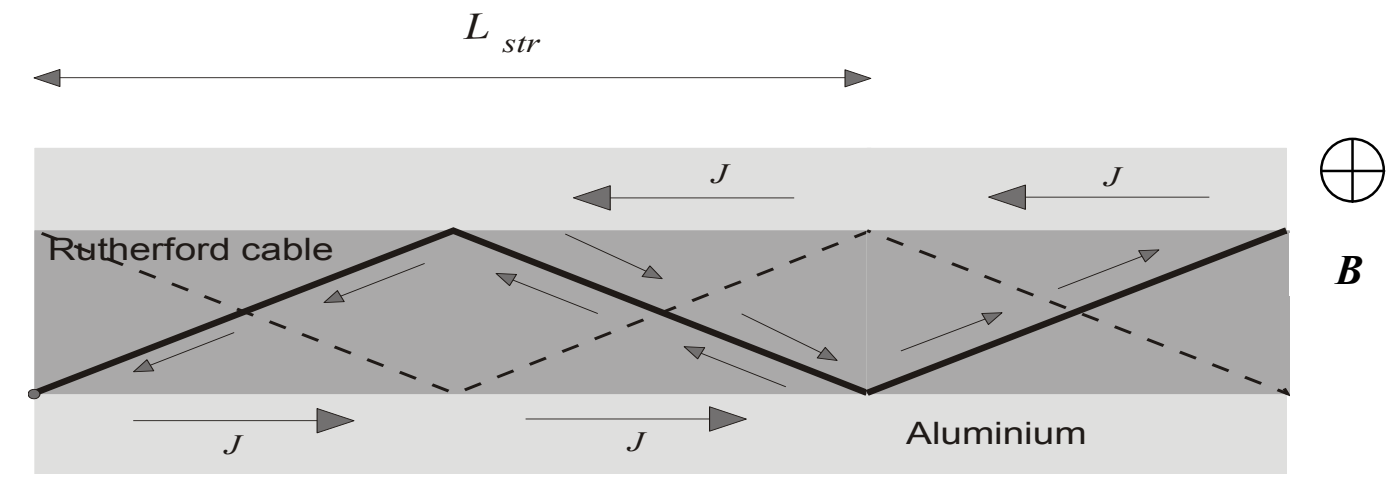

FIGURE 3. Screening currents inside a Rutherford cable well connected to a cladding (stabilizer) with low resistivity, reducing the loss by a factor of two as compared to an ordinary solid bar of Aluminum. 
nection between Aluminum and Rutherford cable is excellent, then the loss due to current through the Aluminum will be dominant. In this case, $\rho_{\text {eff }}=\rho_{A l}$. In general, the dominant loss contribution will determine the effective magnetic field acting on the superconductor. In ATLAS superconductors, the Eddy current loss due to the Cable-to-Aluminum connections is expected to be considerably higher than the intra- and inter strand coupling losses. This is due to the much higher contact resistances, which give an effective resistivity of about $10^{-10} \Omega \mathrm{m}$ or higher, while the resistivity of the Aluminum is in the order of $10^{-11} \Omega \mathrm{m}$. Therefore the screening effect of the Aluminum has an additional important consequence. It screens the Rutherford cable from the external field resulting in a reduction of the coupling current loss.

\section{Magnetization of the Barrel Toroid conductor}

In order to better understand the hysteresis loss contribution, a single strand taken from the Barrel Toroid conductor is investigated using a dedicated setup for measuring the magnetization loss at University of Twente [2]. In figure 4, the magnetization curves versus applied field with amplitudes of 0.4 and $3 \mathrm{~T}$ respectively are shown. The maximum value is reached at the field of full penetration of the filaments, which is $70 \mathrm{mT}$. This value corresponds to a critical current density of $J_{c}=3.9 \cdot 10^{9} \mathrm{~A} / \mathrm{m}^{2}$. The peak value of the magnetization is at $0.129 \mathrm{~A} / \mathrm{m}^{2}$.

Using, magnetic field sweeps between -0.2 and $+0.2 \mathrm{mT}$ with different ramp-rate, the hysteresis loss in the singel strand is determined. Scaling to a complete BT conductor yields a predicted hysteresis loss at zero field rate of $1.49 \mathrm{~mJ} / \mathrm{cycle} / \mathrm{cm}^{3}$.

\section{AC-loss measured in the Barrel Toroid conductor.}

A sample holder including two $0.5 \mathrm{~m}$ long samples of the Barrel Toroid conductor, which are electrically isolated, are placed in a calorimetric AC-loss measurement setup specially developed for calorimetric and inductive cable loss measurments [2].

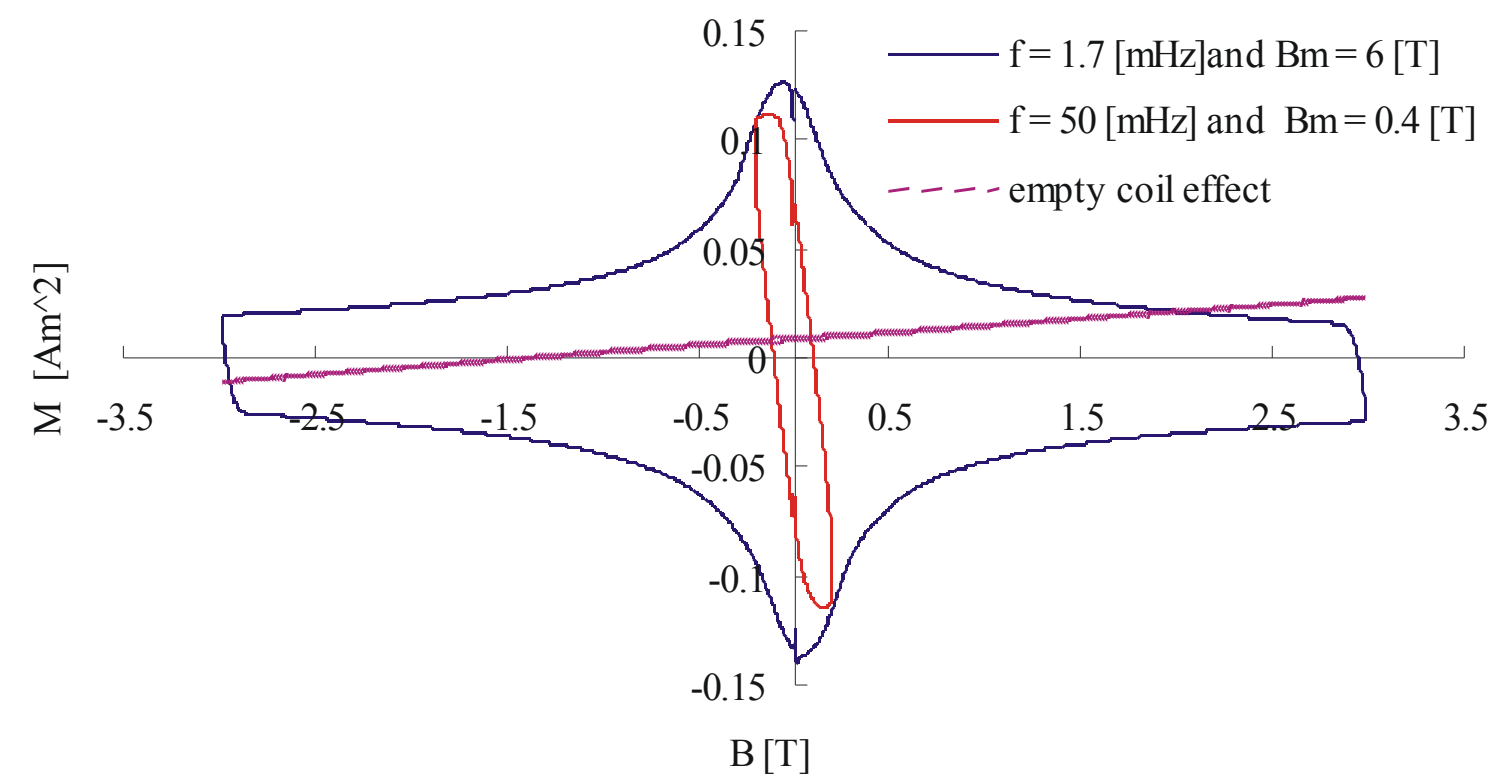

FIGURE 4. Magnetization curves at 0.4 and $3 \mathrm{~T}$ sweeps of the magnetic field measured on a single strand taken from the Barrel Toroid conductor. The curves are corrected for the empty coil effect (inclined line). 


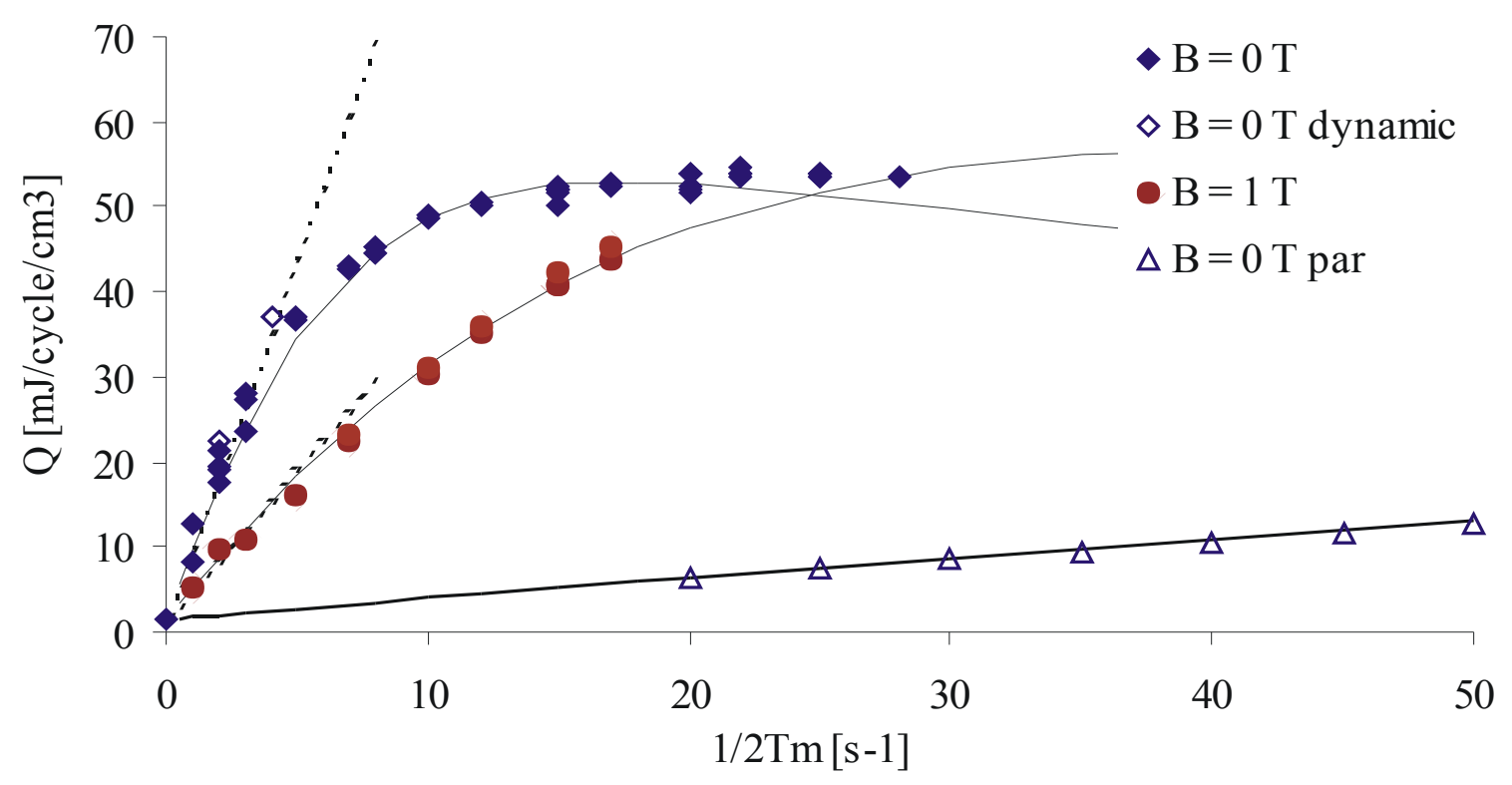

FIGURE 5. Measured (markers) and claculated (lines) AC loss of the BT conductor, $0.4 \mathrm{~T}$ sweep at $0.2 \mathrm{~T}$ peak value, versus 1 / (sweep time) in 0 and $1 \mathrm{~T}$ static background field. Filled markers is perpendicular field, open markers is parallel field.

The external magnet field is varied between -0.2 and $+0.2 \mathrm{~T}$ and the total loss is measured using the calorimetric method and the inductive method as well. The last method is based on a pickup coil wound around the stack of conductors and compensated for the empty coil effect. In order to increase the accuracy, the loss is determined by taking an average value over a number of magnetic field cycles.

In figure 5, the $\mathrm{AC}$ loss in the BT conductor is shown versus the sweep time of the magnetic field with $B_{m}=0.4 \mathrm{~T}$ in a background magnetic field of 0 and $1 \mathrm{~T}$. The varying magnetic field is applied perpendicular (filled symbols) as well as parallel (open symbols) to the conductor. At low frequency, the behavior is linear with a slope proportional to the characteristic time constant of the dominant loss mechanism.

From the slopes of the dashed lines in figure 5, one can extract in the case of perpendicular field the dominant characteristic time constants of $17 \pm 3 \mathrm{~s}$ and $7 \pm 1 \mathrm{~s}$ at $B=0 \mathrm{~T}$ and $B=1 \mathrm{~T}$, respectively. This corresponds to a resistivity of the Aluminum of $1.0 \pm 0.2 \cdot 10^{-11} \Omega \mathrm{m}$ and $2.4 \pm 0.4 \cdot 10^{-12} \Omega \mathrm{m}$. It is found that the data can be described reasonably well with the following expression, shown by lines in figure 5 :

$$
Q_{\text {total }}=\left(Q_{e}+Q_{\text {iccc }}+Q_{\text {hys }} / a b\right) \quad\left[\mathrm{J} / \mathrm{cycle} / \mathrm{m}^{3}\right]
$$

which suggests that the coupling currents between strands and filaments and the eddy currents inside the Rutherford cable are not determining the dominating loss mechanism. This is expected since the Rutherford cable in the conductor center is shielded considerably from the magnetic field by the large amount of high-conductivity stabilizer. The hysteresis loss is taken from the strand measurement.

The resistivity of the Aluminum and the effective resistivity of the Cladding-Cable Coupling Current paths used in the calculations, are listed in the table 1. In the calculation, it is assumed that the Aluminum resistivity $\rho_{A l}$ is constant, which is not correct at low magnetic field and therefore it causes a less accurate result at low magnetic field values. 
TABLE 1. Electrical resistivities of the Aluminum used in the loss calculations.

\begin{tabular}{|cccc|}
\hline $\mathrm{B}[\mathrm{T}]$ & Field direction & Loss $\left[\mathrm{mJ} /\right.$ cycle $\left./ \mathrm{cm}^{3}\right]$ & Resistivity $\left[\mathrm{x} 10^{-11} \Omega \mathrm{m}\right]$ \\
\hline 0 & Perpendicular & $\mathrm{Q}_{\mathrm{e}}$ & 1.0 \\
0 & Perpendicular & $\mathrm{Q}_{\mathrm{cccc}}$ & 30 \\
\hline 1 & Perpendicular & $\mathrm{Q}_{\mathrm{e}}$ & 2.4 \\
1 & Perpendicular & $\mathrm{Q}_{\mathrm{cccc}}$ & 30 \\
\hline 0 & Parallel & $\mathrm{Q}_{\mathrm{e}}$ & 15 \\
0 & Parallel & $\mathrm{Q}_{\mathrm{cccc}}$ & 30 \\
\hline
\end{tabular}

\section{Dynamic measurements of the AC-loss in Al-stabilized NbTi/Cu superconductors}

In the case of a loss calculation for the complete magnet, the loss has to be scaled to the local magnetic field across the windings. In stead another method may be used.

The losses can alos be measured by directly recording the power loss over a large magnetic field interval, which then enables to calculate the total AC-loss in a magnet by using the power loss found for a given magnetic field sweep that corresponds to the local field in the coil. In the AC-loss measurement set-up, the power loss is determined using a varying external magnetic fields up to $2 \mathrm{~T}$. The helium gas that evaporates is detected with some delay, as the gas has to flow first through the pipe work before reaching the calibrated flow meter. The system behaves as a first order process with a characteristic time constant of $20 \mathrm{~s}$ at $0.3 \mathrm{~kW}$ and $35 \mathrm{~s}$ below $100 \mathrm{~W}$.

The measured power loss and the corrected power loss, taking the Laplace transformations, are presented in figure 6. After ramping the magnetic field down to $-2 \mathrm{~T}$, the magnetic field is increased with $4 \mathrm{mT} / \mathrm{s}$. The corrected power loss shows a higher loss until the penetration field is reached at $+0.1 \mathrm{~T}$. At this point the screening currents are reversed and the internal magnetic field is zero. At this point the resistivity of the Aluminum is minimum and the AC-losses reach the maximum value. If the magnetic field is increased to

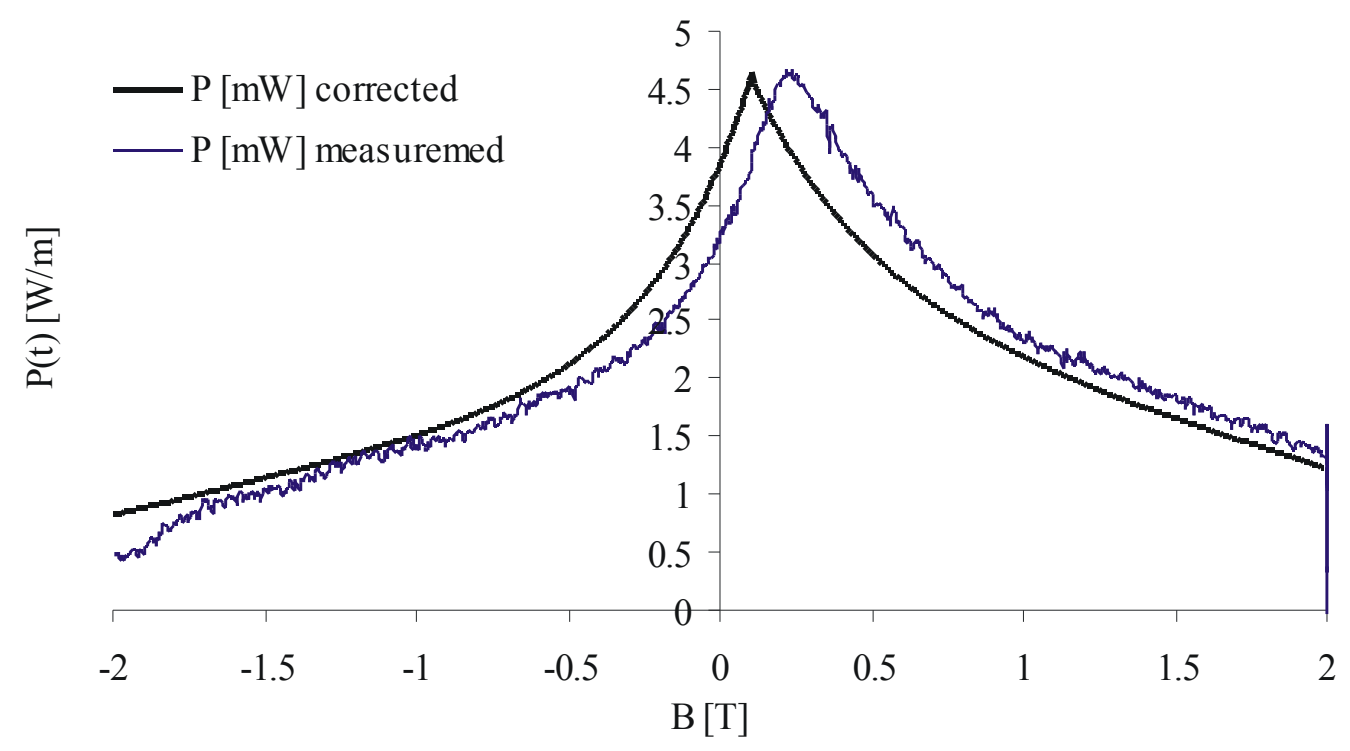

FIGURE 6. Power loss versus magnetic field of the Central Solenoid conductor. The measured curve has to be corrected for the characteristics of the measurement set-up. The applied magnetic field starts at $\mathrm{B}=-2 \mathrm{~T}$ and is increased with a constant ramp-rate of $4 \mathrm{mT} / \mathrm{s}$ up to $2 \mathrm{~T}$. 
$2 \mathrm{~T}$ the corrected power loss becomes almost equal to the measured losses, as the change is small compared to the characteristic time constant of the experimental set-up.

The AC-loss of the Solenoid conductor is determined in the calorimetric set-up also in the same way as for the BT conductor. The power loss measurement is compared quantitatively by integrating over the field sweep of -0.2 to $+0.2 \mathrm{~T}$, which yields $6.4 \mathrm{~mJ} / \mathrm{cycle} / \mathrm{cm}^{3}$ with a period time of $200 \mathrm{~s}$.

As shown in figure 6 for the Solenoid conductor, also for the BT conductor the power loss measurement is performed at ramp-rates of $3.2 \mathrm{mT} / \mathrm{s}$ and $1.6 \mathrm{mT} / \mathrm{s}$ corresponding to period times 250 and $500 \mathrm{~s}$. The results are depicted in figure $5(\mathrm{~B}=0 \mathrm{~T}$ dynamic) and are in good agreement with other data. In order to determine the losses inside magnets, this measurement technique can give the total losses over a large magnetic field ranch using only one measurement.

\section{Conclusions}

The AC-losses of the Barrel Toroid and the Central Solenoid conductors used in the ATLAS Magnet System is measured in an external varying magnetic field is studied. Magnetic field is applied perpendicular as well as parallel to the conductor wide surface. The results are compared to the AC-loss theory taken into account the diffusion in the large size conductors.

It is found that the main losses are due to hysteresis loss, the eddy current loss inside the Aluminum cladding and the cable-cladding coupling current loss (CCCC). The case is similar to high-temperature superconductors like a layer of $\mathrm{BiSrCaCuO}$ filaaments surrounded by Ag-alloy sheet.

Near zero tesla, the calculations become less accurate as the resistivity of pure Aluminum is strongly dependent on the magnetic field value. Despite this effect, the results of the model adapted for this case and the measured data are in satisfactory agreement.

The power loss due to an external magnetic field sweep between -2 and 2 tesla at constant ramp-rate is determined for both conductors and compared to the AC-loss measurement results.

It was demonstrated that the total AC loss over a large range of magnetic field amplitudes could be determined when performing very a single power loss measurement.

\section{REFERENCES}

1. H.H.J. ten Kate, "Superconducting Magnet System for the ATLAS Detector at CERN", IEEE Transactions On Applied Superconductivity, Volume 10, N1 p. 347, 2000.

2. A.P. Verwey, A. den Ouden, B. Sachse and H.H.J. ten Kate, Advances in Cryogenic Engineering, 1994.

3. M.N. Wilson, "Superconducting Magnets", Clarendon Press, Oxford 1983.

4. K.V. Namjoshi and P.P. Biringer, "Low-Frequency Eddy-Current Loss Estimation in Long Conductors by using the Moment of Inertia of Cross Sections", IEEE Transactions on Magnetics Volume 24, No 5, p.2181-2185, September 1988.

5. A.M. Campbell, Cryogenics Nov., 1982, p651.

6. W.J. Carr, Jr. "Conductivity, permeability, and dielectric constant in a multifilament superconductor", Journal of Applied Physics, Volume 46, No. 9, September 1975. 